\title{
Modified phacoemulsification plus goniosynechialysis compared with conventional surgery for cataract and glaucoma
}

\author{
YURONG TANG ${ }^{1}$, JILIN TAN $^{1}$, XIAOHONG ZHOU $^{1}$ and XIANGJI LI $^{2}$ \\ ${ }^{1}$ Department of Cataract and Glaucoma, Chongqing Aier Mega Eye Hospital, \\ Chongqing 400060; ${ }^{2}$ Department of Ophthalmology, The Third Affiliated Hospital \\ of Chongqing Medical University, Chongqing 401120, P.R. China
}

Received September 6, 2019; Accepted November 1, 2019

DOI: $10.3892 / \mathrm{etm} .2019 .8182$

\begin{abstract}
Optic nerve damage and visual impairment caused by glaucoma affect 66.8 million people worldwide, and causing bilateral blindness in 6.7 million people. Surgery is the main method for the treatment of cataract with glaucoma. In recent years, clinicians have increasingly paid attention to and applied phacoemulsification and intraocular lens implantation combined with goniosynechialysis for the treatment of cataract with angle-closure glaucoma. However, for patients with complicated cataract, the high ultrasonic energy of traditional phacoemulsification can largely damage the corneal endothelium. Modified phacoemulsification (lower ultrasonic energy) and intraocular lens implantation have now achieved certain efficacy. The efficacy and safety of modified phacoemulsification plus goniosynechialysis compared with conventional surgery for cataract and glaucoma was investigated. A total of 125 patients who underwent goniosynechialysis combined with phacoemulsification and intraocular lens implantation were enrolled in the control group, while 179 patients treated by modified phacoemulsification and intraocular lens implantation combined with goniosynechialysis were enrolled in the research group. The visual acuity and intraocular pressure were observed before and 6 months after surgery in both groups, and the incidence of complications was analyzed. After treatment, there were more patients with visual acuity of 0.2-0.4 and $>0.4$ in the research group than in the control group $(\mathrm{P}<0.05)$. The incidence of corneal edema and anterior chamber inflammation was lower in the research group than in the control group (both $\mathrm{P}<0.05$ ), while the preoperative and postoperative intraocular pressure, central anterior chamber depth, angle-opening distance, and peripheral iridocorneal adhesions were not significantly different between the two
\end{abstract}

Correspondence to: Dr Xiangji Li, Department of Ophthalmology, The Third Affiliated Hospital of Chongqing Medical University, 1 Shuanghu Branch Road, Yubei, Chongqing 401120, P.R. China E-mail: jef59k@163.com; lixiangji97ghv@163.com

Key words: cataract and glaucoma, modified phacoemulsification, goniosynechialysis, clinical efficacy, safety groups (all $\mathrm{P}>0.05$ ). Modified phacoemulsification and intraocular lens implantation plus goniosynechialysis for cataract with glaucoma can better improve the visual acuity, as well as effectively reduce corneal edema and anterior chamber inflammation.

\section{Introduction}

Optic nerve damage and visual impairment caused by glaucoma as a global health problem, is the second most common cause of blindness in the world, affecting 66.8 million people and causing bilateral blindness in 6.7 million people. Besides, as the population ages, its incidence shows a further upward trend $(1,2)$. There are two types of glaucoma: angle-closure glaucoma and open-angle glaucoma. Cataract is a major cause of angle-closure glaucoma, because intumescent cataract can lead to acute closure of patient's angle, then induce glaucoma, which is a very common phenomenon in clinic $(3,4)$. Surgery is the main treatment for cataract with glaucoma.

To date, the most commonly used operating methods in clinic are trabeculectomy, phacoemulsification and intraocular lens implantation, among which trabeculectomy is the most classic one (5). However, trabeculectomy can lead to a high incidence of postoperative complications despite the continuous improvement of trabeculectomy technique and the combination with lens removal, which could effectively prevent the peripheral iridocorneal adhesions. Once the adhesions develop, they remain and worsen the disease condition $(6,7)$. In recent years, clinicians have increasingly paid attention to and applied phacoemulsification and intraocular lens implantation combined with goniosynechialysis for the treatment of cataract with angle-closure glaucoma. Studies have reported that phacoemulsification and intraocular lens implantation combined with goniosynechialysis can effectively reduce intraocular pressure, without developing complications that traditional glaucoma surgery brings, such as low intraocular pressure, macular edema or recurrent angle closure $(8,9)$. However, traditional phacoemulsification and intraocular lens implantation also have some shortcomings. For patients with complicated cataract, the high ultrasonic energy of traditional phacoemulsification largely damages the corneal endothelium (10). Modified phacoemulsification (lower ultrasonic energy) and intraocular lens implantation 
have now achieved certain efficacy in patients with cataract and high myopia (11).

The present study retrospectively analyzed the application of modified phacoemulsification and intraocular lens implantation combined with goniosynechialysis for the treatment of cataract with glaucoma, to evaluate the therapeutic effect and safety, and to provide further guidance for clinical treatment.

\section{Patients and methods}

Subjects. Data of 304 cases of cataract with glaucoma admitted to The Third Affiliated Hospital of Chongqing Medical University from April 2017 to September 2018 were retrospectively analyzed. Patients were aged 30-70 years. They were enrolled into two groups according to different treatment methods: 179 patients treated with modified phacoemulsification and intraocular lens implantation combined with goniosynechialysis were included in the study group; 125 patients treated with phacoemulsification and intraocular lens implantation combined with goniosynechialysis were included in the control group. This study was approved by the Ethics Committee of The Third Affiliated Hospital of Chongqing Medical University, and the informed consent form was obtained from all the patients and their families by informing them via telephone or letter.

Patients were eligible if they had glaucoma that met the diagnostic criteria of the Expert Consensus on Diagnosis and Treatment of Primary Glaucoma (2014) developed by the Ophthalmology Branch, Chinese Medical Association (12); had acute attack; had only one eye involved; had complete clinical data; had no past medical history of tumor, glaucoma, or any eye surgery including visual correction. Patients were excluded if they had open angle glaucoma, chronic angle-closure glaucoma, or secondary glaucoma; had surgical contraindications; had abnormal bleeding, other eye diseases, diabetes, systemic inflammatory response, autoimmune diseases, or serious defects in cardiopulmonary function; had incomplete medical records; were pregnant or in lactation.

Therapeutic methods. All patients were given pilocarpine nitrate eye drops $1 \mathrm{~h}$ before surgery to reduce intraocular pressure. If necessary, $250 \mathrm{ml}$ of $20 \%$ mannitol was given to reduce intraocular pressure, which was maintained at $\leq 25 \mathrm{mmHg}$.

In the control group, phacoemulsification and intraocular lens implantation combined with goniosynechialysis were performed. After topical and peribulbar anesthesia with oxybuprocaine hydrochloride and lidocaine, a tunnel incision was made along the transparent corneal edge at direction of 11 o'clock. Then, $0.2 \mathrm{ml}$ of viscoelastic agent was injected into the anterior chamber to perform continuous curvilinear capsulorhexis. The physiological saline was used for hydrodissection, and the phacoemulsification apparatus was used to emulsify the lens nucleus, and the perfusion/resorption system was used to remove the residual cortex. Next, $0.3 \mathrm{ml}$ of viscoelastic agent was injected to enlarge the angle. The intraocular lens in appropriate size was placed in the capsule before the goniosynechialysis was conducted. The root of the iris was pressed backward $360^{\circ}$ along the angle with the use of sodium hyaluronate, and the adhesions of the angle were separated twice. After that, the residue such as viscoelastic agent was extracted, the incision of transparent corneal was hydrated, and carefully observed for aqueous humor leakage. After injection of $2 \mathrm{mg}$ of dexamethasone under the bulbar conjunctiva, the eye was dressed with sterile gauze.

In the research group, modified phacoemulsification and intraocular lens implantation combined with goniosynechialysis were performed. After topical and peribulbar anesthesia with oxybuprocaine hydrochloride and lidocaine, a tunnel incision was made along the transparent corneal edge at direction of 11 o'clock. Then, $0.2 \mathrm{ml}$ of viscoelastic agent was injected into the anterior chamber to perform continuous curvilinear capsulorhexis. Physiological saline was used for hydrodissection, and the phacoemulsification apparatus emulsified the lens nucleus in a mode of high negative pressure, low energy, and low discharge to assist the nucleus fragmentation. The chopper was placed in the appropriate site for nucleus fragmentation. The perfusion/resorption system was then used to remove the residual cortex. Next, $0.3 \mathrm{ml}$ of viscoelastic agent was injected to enlarge the angle. An intraocular lens of appropriate size was placed in the capsule before the goniosynechialysis was conducted. The root of the iris was pressed backward $360^{\circ}$ along the angle with the use of sodium hyaluronate, and the adhesions of the angle were separated twice. After that, the residue such as viscoelastic agent was extracted, and the incision of transparent corneal was hydrated, and the aqueous humor leakage carefully observed. After injection of $2 \mathrm{mg}$ of dexamethasone under the bulbar conjunctiva, the eye was dressed with sterile gauze.

Outcome measures. All patients were followed 6 months after surgery. The visual acuity, intraocular pressure, central anterior chamber depth, angle-opening distance, and peripheral iridocorneal adhesions were observed before and after surgery. The incidence of complications was compared between the two groups.

Statistical analyses. SPSS19.0 (Asia Analytics Formerly SPSS China) was used for statistical processing. The count data were expressed as rate, which was compared using $\chi^{2}$ test, and the comparison between before and after treatment within group was performed using McNemar's test. The measurement data, which were expressed as mean \pm standard deviation, compared between two groups using independent sample t-test, and compared between before and after treatment within group using paired t-test. $\mathrm{P}<0.05$ was considered to indicate a statistically significant difference.

\section{Results}

Baseline data. In the control group, there were 125 patients, including 72 males $(57.60 \%)$ and 53 females (42.40\%), aged $52.8 \pm 8.5$ years. In the research group, there were 179 patients, including 107 males (59.78\%) and 72 females (40.22\%), aged $54.6 \pm 9.1$ years. There was no significant difference in sex, age, Lens Opacities Classification System II grading and Scheie classification between the two groups (all P>0.05) (Table I).

Comparison of changes in visual acuity. Before surgery, the visual acuity was not statistically different between the two 
Table I. Baseline data.

\begin{tabular}{|c|c|c|c|c|}
\hline Factors & Control group $(n=125)$ & Research group $(\mathrm{n}=179)$ & $\chi^{2} / \mathrm{t}$ value & P-value \\
\hline $\operatorname{Sex}(n, \%)$ & & & 0.144 & 0.704 \\
\hline Male & $72(57.60)$ & $107(59.78)$ & & \\
\hline Female & $53(42.40)$ & $72(40.22)$ & & \\
\hline Age (year) & $52.8 \pm 8.5$ & $54.6 \pm 9.1$ & 1.789 & 0.075 \\
\hline LOCS II grading $(\mathrm{n}, \%)$ & & & 1.125 & 0.570 \\
\hline II & $37(29.60)$ & $47(26.26)$ & & \\
\hline III & $74(59.20)$ & $105(58.66)$ & & \\
\hline IV & $14(11.20)$ & $27(15.08)$ & & \\
\hline Diseased eye $(\mathrm{n}, \%)$ & & & 0.712 & 0.399 \\
\hline Left eye & $62(49.60)$ & $80(44.69)$ & & \\
\hline Right eye & $63(50.40)$ & $99(55.31)$ & & \\
\hline Scheie's classification $(\mathrm{n}, \%)$ & & & 0.282 & 0.869 \\
\hline N II & $25(20.00)$ & $40(22.35)$ & & \\
\hline N III & $34(27.20)$ & $49(27.37)$ & & \\
\hline N IV & $66(52.80)$ & $90(50.28)$ & & \\
\hline
\end{tabular}

LOCS, Lens Opacities Classification System.

Table II. Changes in visual acuity.

\begin{tabular}{|c|c|c|c|c|}
\hline Parameters & Control group $(n=125)$ & Research group (n=179) & $\chi^{2}$ value & $\mathrm{P}$-value \\
\hline Before treatment & & & 0.928 & 0.629 \\
\hline$<0.1$ & $44(35.20)$ & $66(36.87)$ & & \\
\hline $0.1-0.2$ & $60(48.00)$ & $90(50.28)$ & & \\
\hline $0.2-0.4$ & $21(16.80)$ & $23(12.85)$ & & \\
\hline$>0.4$ & $0(0.00)$ & $0(0.00)$ & & \\
\hline After treatment & & & 11.192 & 0.011 \\
\hline$<0.1$ & $11(8.80)$ & $7(3.91)$ & & \\
\hline $0.1-0.2$ & $41(32.80)$ & $46(25.70)$ & & \\
\hline $0.2-0.4$ & $36(28.80)$ & $61(34.08)$ & & \\
\hline$>0.4$ & $37(29.60)$ & $65(36.31)$ & & \\
\hline$\chi^{2}$ value & 64.322 & 144.111 & & \\
\hline P-value & $<0.001$ & $<0.001$ & & \\
\hline
\end{tabular}

groups $(\mathrm{P}>0.05)$, while after treatment, there was a statistically significant difference in the visual acuity between the two groups $(\mathrm{P}<0.05)$. Further McNemar's test showed that there were more patients with visual acuity of $0.2-0.4$, and $>0.4$ in the research group than in the control group (both $\mathrm{P}<0.05$ ) (Table II).

Comparison of changes in intraocular pressure. Before surgery, there was no significant difference in the intraocular pressure between the two groups $(\mathrm{P}>0.05)$. After surgery, the intraocular pressure was decreased in both groups (both $\mathrm{P}<0.001$ ) (Fig. 1).

Comparison of changes in central anterior chamber depth. Before surgery, there was no significant difference in the central anterior chamber depth between the two groups $(\mathrm{P}>0.05)$. After surgery, the central anterior chamber depth of the two groups both increased to different degrees (both $\mathrm{P}<0.001$ ) (Fig. 2).

Comparison of changes in angle-opening distance. Before surgery, there was no significant difference in the angleopening distance between the two groups $(\mathrm{P}>0.05)$. After surgery, the angle-opening distance of the two groups both increased to different degrees (both $\mathrm{P}<0.001$ ) (Fig. 3).

Comparison of changes in peripheral iridocorneal adhesions. The peripheral iridocorneal adhesions were reduced after surgery as compared with before surgery in both groups, while there was no difference in preoperative or postoperative 


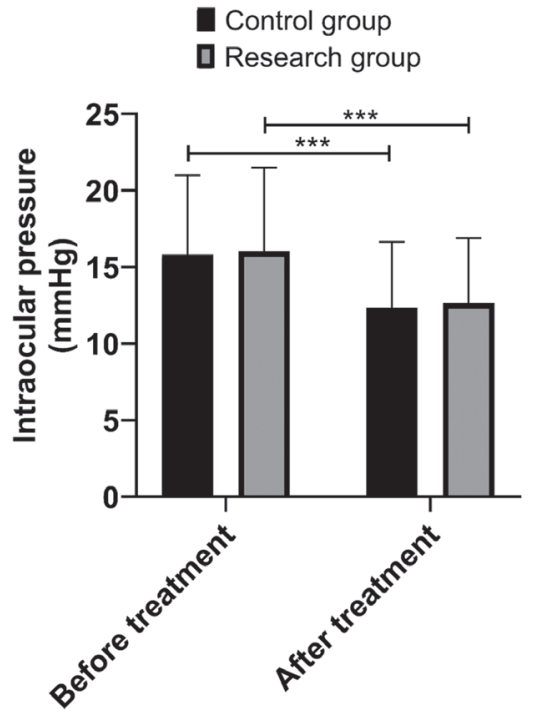

Figure 1. Changes in intraocular pressure. ${ }^{* * * *} \mathrm{P}<0.001$.

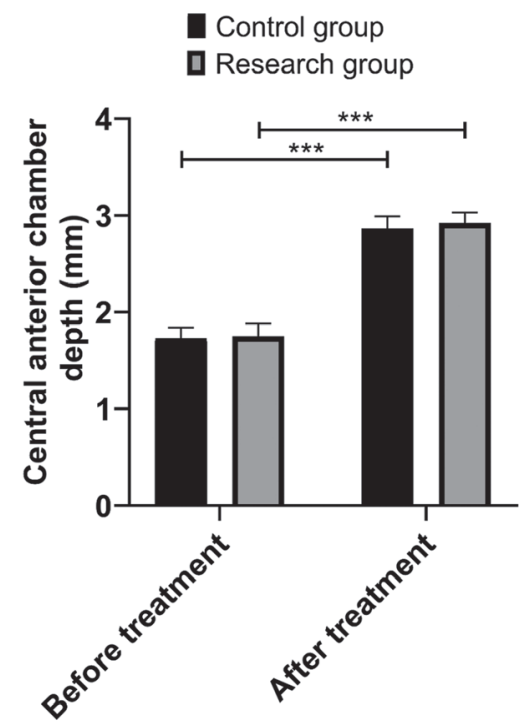

Figure 2. Changes in central anterior chamber depth. ${ }^{* * *} \mathrm{P}<0.001$.

peripheral iridocorneal adhesions between the two groups (both $\mathrm{P}>0.05$ ) (Table III).

Comparison of complications. The total incidence of complications in the research group was significantly lower than that in the control group $(\mathrm{P}<0.05)$. Moreover, the incidence of corneal edema and anterior chamber inflammation was significantly lower in the research group than in the control group (both $\mathrm{P}<0.05$ ). However, there was no significant difference in the incidence of hyphema and fibrinous exudate in the anterior chamber between the two groups (both $\mathrm{P}>0.05$ ) (Table IV).

\section{Discussion}

Angle-closure glaucoma is predominantly caused by the blocking of the aqueous humor outflow due to the closure of the angle, resulting in increased intraocular pressure, thereby

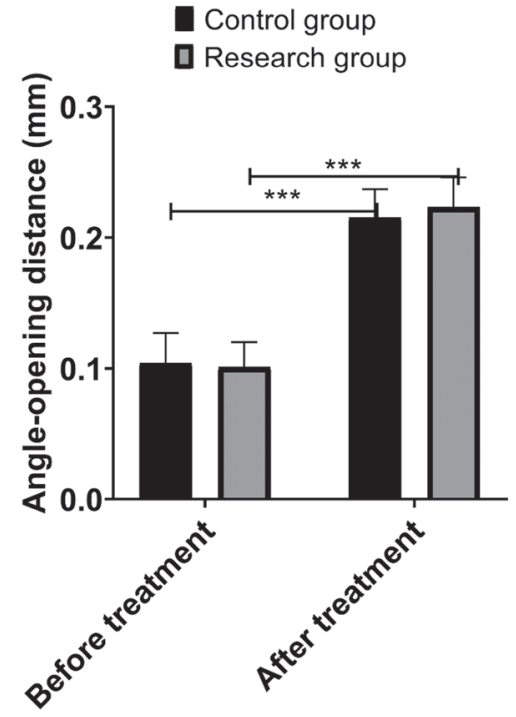

Figure 3. Changes in angle-opening distance. ${ }^{* * *} \mathrm{P}<0.001$

causing optic nerve damage and progressive visual impairment. It is estimated that the number of glaucoma patients worldwide will reach 79.6 million by $2020(13,14)$. The main principle of clinical treatment for angle-closure glaucoma is to relieve the closure of the angle and the adhesions. Goniosynechialysis is a surgical method that removes the peripheral iridocorneal adhesions from the angle wall to reconstruct the function of the trabeculae, which can effectively reduce the intraocular pressure and peripheral iridocorneal adhesions in patients with angle-closure glaucoma $(15,16)$. Furthermore, in patients with glaucoma caused by cataract, the treatment should also give consideration to glaucoma. It has been reported that combined surgeries were more effective than surgery for cataract alone in reducing intraocular pressure and could reduce the administration of postoperative anti-glaucoma drugs (17).

The two groups in this study were comparable as there was no significant difference in the baseline data between the two groups. The results of this study showed that the improvement of visual acuity in the research group was more significant than that in the control group, while the improvement of intraocular pressure, central anterior chamber depth, open-angle distance, and the iridocorneal adhesions were not significantly different between the two groups. However, the research group showed lower incidence of complications than the control group, mainly including lower incidence of corneal edema and anterior chamber inflammation. The reduction of phacoemulsification energy in cataract surgery can decrease the postoperative corneal swelling and endothelial cell loss (18). Jacob (19) reported the advantages of assisted nucleus fragmentation in phacoemulsification. The advantages were effective reduction of ultrasound energy, endothelial damage, and eye stress. Ianchulev et al (20) also reported lower rate of endothelial cell loss caused by combined nucleus fragmentation than by phacoemulsification alone. However, the present study lacks data on endothelial cell detection as this is a retrospective analysis, so further prospective studies or randomized controlled studies to supplement our results are required.

In addition, the intraocular pressure after goniosynechialysis depends on the function of the trabecular meshwork. 
Table III. Changes in peripheral iridocorneal adhesions.

\begin{tabular}{|c|c|c|c|c|}
\hline Parameters & Control group $(n=125)$ & Research group $(n=179)$ & $\chi^{2}$ value & P-value \\
\hline Before treatment & & & 0.696 & 0.706 \\
\hline$<180^{\circ}$ & $44(35.20)$ & $58(32.40)$ & & \\
\hline $180^{\circ}-270^{\circ}$ & $40(32.00)$ & $54(30.17)$ & & \\
\hline$>270^{\circ}$ & $41(32.80)$ & $67(37.43)$ & & \\
\hline After treatment & & & 2.049 & 0.152 \\
\hline$<180^{\circ}$ & $95(76.00)$ & $148(82.68)$ & & \\
\hline $180^{\circ}-270^{\circ}$ & $30(24.00)$ & $31(17.32)$ & & \\
\hline$>270^{\circ}$ & $0(0.00)$ & $0(0.00)$ & & \\
\hline$\chi^{2}$ value & 61.141 & 112.544 & & \\
\hline P-value & $<0.001$ & $<0.001$ & & \\
\hline
\end{tabular}

Table IV. Incidence of complications (n, \%).

\begin{tabular}{|c|c|c|c|c|}
\hline Factors & Control group $(n=125)$ & Research group $(n=179)$ & $\chi^{2}$ value & P-value \\
\hline Corneal edema & $16(12.80)$ & $9(5.03)$ & 5.891 & 0.015 \\
\hline Hyphema & $3(2.40)$ & $1(0.56)$ & 1.922 & 0.166 \\
\hline Fibrinous exudate in the anterior chamber & $2(1.60)$ & $3(1.68)$ & 0.003 & 0.959 \\
\hline Anterior chamber inflammation & $7(5.60)$ & $2(1.12)$ & 5.148 & 0.023 \\
\hline In total & $28(22.40)$ & $15(8.38)$ & 11.913 & 0.001 \\
\hline
\end{tabular}

Long-term peripheral iridocorneal adhesions may cause irreversible damage on the trabecular meshwork. Therefore, the duration of peripheral iridocorneal adhesions must be considered before goniosynechialysis $(16,21)$. This was also confirmed by Lee et al (22) who reported similar therapeutic effects of phacoemulsification and intraocular lens implantation combined with goniosynechialysis and of phacoemulsification and intraocular lens implantation alone in patients with primary angle-closure glaucoma and favorable preoperative intraocular pressure. Different preoperative intraocular pressure may be the reason for the different therapeutic effects of goniosynechialysis in some studies. There are some limitations in this study. Retrospective analysis can inevitably result in some selection bias. Therefore, the results of this study still need further confirmation by clinical randomized controlled trials.

In summary, modified phacoemulsification and intraocular lens implantation combined with goniosynechialysis for the treatment of cataract with glaucoma can better improve the visual acuity, and reduce corneal edema and anterior chamber inflammation. Since the intraocular pressure after goniosynechialysis depends on the function of the trabecular meshwork, the possible duration of peripheral iridocorneal adhesions should be considered before goniosynechialysis, so as to improve the iridocorneal adhesions.

\section{Acknowledgements}

Not applicable.

\section{Funding}

No funding was received.

\section{Availability of data and materials}

The datasets used and/or analyzed during the current study are available from the corresponding author on reasonable request.

\section{Authors' contributions}

YT and JT performed experimental operation; JT and XZ contributed to experimental data (pathological pictures); YT and XZ contributed to data statistics; YT contributed to data analysis; YT wrote the manuscript; YT contributed to the study design. XL guided the study. All authors read and approved the final manuscript.

\section{Ethics approval and consent to participate}

The study was approved by the Ethics Committee of The Third Affiliated Hospital of Chongqing Medical University, China. Informed consent form was obtained from the patients and their families. Informed consent statement: Patients were not required to give informed consent to the study because the analysis used anonymous clinical data that were obtained after each agreed to treatment by written consent. 


\section{Patient consent for publication}

Not applicable.

\section{Conflict of interest}

The authors declare that they have no competing interests.

\section{References}

1. Henson DB and Thampy R: Preventing blindness from glaucoma. BMJ 331: 120-121, 2005.

2. Chang LS, Lee HC, Tsai YC, Shen LS, Li CL, Liu SF and Kuo HC: Decreased incidence of glaucoma in children with asthma using inhaled corticosteroid: A cohort study. Oncotarget 8: 105463$105471,2017$.

3. Mishra C, Sundar T, Sanjeev S, Babu N and Kim R: Hydrochlorothiazide sensitised acetazolamide induced bilateral angle closure glaucoma with choroidal detachment after cataract surgery. J Clin Diagn Res 12: 4-6, 2018.

4. Li HJ, Xuan J, Zhu XM and Xie L: Comparison of phacotrabeculectomy and sequential surgery in the treatment of chronic angle-closure glaucoma coexisted with cataract. Int J Ophthalmol 9: 687-692, 2016.

5. Morgan WH and Yu DY: Surgical management of glaucoma: A review. Clin Exp Ophthalmol 40: 388-399, 2012.

6. Lin HZ, Huang CT and Lee YC: A blood clot hanging in the anterior chamber due to delayed suprachoroidal hemorrhage after trabeculectomy. Ci Ji Yi Xue Za Zhi 28: 73-75, 2016 (In Chinese).

7. Solanki M, Kumar A, Upadhyay A and Kumar K: Viscoelasticaugmented trabeculectomy: A newer concept. Indian J Ophthalmol 65: 705-711, 2017.

8. Panse K, Le C, Hubbell M and Ayyala RS: Surgical outcomes of phacoemulsification/goniosynechialysis with and without endocyclophotocoagulation in patients with chronic angle closure glaucoma. Indian J Ophthalmol 67: 366-370, 2019.

9. Liu H: Phacoemulsification combined with goniosynechialysis treating cataract with acute angle-closure glaucoma. Int Eye Sci 18: 1643-1647, 2018

10. Chen H, Lin H, Chen W, Zhang B, Xiang W, Li J, Chen W and Liu Y: Femtosecond laser combined with non-chopping rotation phacoemulsification technique for soft-nucleus cataract surgery: A prospective study. Sci Rep 6: 18684, 2016.

11. Akal A, Goncu T, Cakmak SS, Yuvaci I, Atas M, Demircan S and Yilmaz OF: Evaluation of early results of quick-chop phacoemulsification in the patients with high myopic cataract. Int J Ophthalmol 7: 828-831, 2014.
12. Glaucoma Group: Ophthalmology Branch, Chinese Medical Association: Expert Consensus on Diagnosis and Treatment of Primary Glaucoma. Chin J Ophthalmol 44: 862-863, 2008.

13. Rao A, Padhy D, Das G and Sarangi S: Evolving paradigms in classification of primary angle closure glaucoma. Semin Ophthalmol 32: 228-236, 2017.

14. Quigley HA and Broman AT: The number of people with glaucoma worldwide in 2010 and 2020. Br J Ophthalmol 90: 262-267, 2006

15. Zhao XJ, Yang XX, Fan YP, Li BH and Li Q: Comparison of combined phacoemulsification, intraocular lens implantation, and goniosynechialysis with phacotrabeculectomy in the treatment of primary angle-closure glaucoma and cataract. Asia Pac J Ophthalmol (Phila) 2: 286-290, 2013.

16. Rodrigues IA, Alaghband P, Beltran Agullo L, Galvis E, Jones S, Husain R and Lim KS: Aqueous outflow facility after phacoemulsification with or without goniosynechialysis in primary angle closure: A randomised controlled study. Br J Ophthalmol 101: 879-885, 2017.

17. Zhang ML, Hirunyachote P and Jampel H: Combined surgery versus cataract surgery alone for eyes with cataract and glaucoma. Cochrane Database Syst Rev 7: CD008671, 2015.

18. Takács AI, Kovács I, Miháltz K, Filkorn T, Knorz MC and Nagy ZZ: Central corneal volume and endothelial cell count following femtosecond laser-assisted refractive cataract surgery compared to conventional phacoemulsification. J Refract Surg 28: 387-391, 2012.

19. Jacob S: Different methods of chopping in cataract surgery. Curr Opin Ophthalmol 30: 25-38, 2019.

20. Ianchulev T, Chang DF, Koo E, MacDonald S, Calvo E, Tyson FT, Vasquez A and Ahmed IIK: Microinterventional endocapsular nucleus disassembly: Novel technique and results of first-inhuman randomised controlled study. Br J Ophthalmol 103: 176-180, 2019.

21. Lai JS, Tham CC and Chan JC: The clinical outcomes of cataract extraction by phacoemulsification in eyes with primary angle-closure glaucoma (PACG) and co-existing cataract: A prospective case series. J Glaucoma 15: 47-52, 2006.

22. Lee CK, Rho SS, Sung GJ, Kim NR, Yang JY, Lee NE, Hong S and Kim CY: Effect of goniosynechialysis during phacoemulsification on IOP in patients with medically well-controlled chronic angle-closure glaucoma. J Glaucoma 24: 405-409, 2015.

This work is licensed under a Creative Commons Attribution-NonCommercial-NoDerivatives 4.0 International (CC BY-NC-ND 4.0) License. 